To be published in Phys. Rev. Lett.

\title{
Electronic structure of intentionally disordered AlAs/GaAs superlattices
}

\author{
Kurt A. Mäder, Lin-Wang Wang, and Alex Zunger \\ National Renewable Energy Laboratory, Golden, CO 80401
}

(Received: 5 October 1994)

\begin{abstract}
We use realistic pseudopotentials and a plane-wave basis to study the electronic structure of non-periodic, three-dimensional, 2000-atom $(\mathrm{AlAs})_{n} /(\mathrm{GaAs})_{m}(001)$ superlattices, where the individual layer thicknesses $n, m \in\{1,2,3\}$ are randomly selected. We find that while the band gap of the equivalent $(n=m=2)$ ordered superlattice is indirect, random fluctuations in layer thicknesses lead to a direct gap in the planar Brillouin zone, strong wavefunction localization along the growth direction, short radiative lifetimes, and a significant band-gap reduction, in agreement with experiments on such intentionally grown disordered superlattices.
\end{abstract}

PACS numbers: 73.20.Dx,78.66.- $-\mathrm{w}, 71.50 .+\mathrm{t}$

Typeset using REVTEX 
Ordered semiconductor superlattices - produced routinely by epitaxial crystal growth techniques - are widely recognized for their unique electronic and optical properties [1]. To tailor the electronic properties (e.g., through band folding) one aims at growing ordered superlattices (o-SL) with definite values of the thicknesses $n$ and $m$ in $(A)_{n} /(G)_{m}$. One could, however, deliberately grow a disordered superlattice (d-SL) [2, 3], where the individual layer thicknesses $n, m, n^{\prime}, m^{\prime}, n^{\prime \prime}, m^{\prime \prime}, \ldots$ are selected at random according to a given probability distribution $p_{\alpha}(n)(\alpha=A, G)$. While the electronic structure of an o-SL is characterized by extended states and the formation of mini-bands, a truly one-dimensional disordered system (described, e.g., by the Anderson model) shows carrier localization and absence of dispersion [4.5]. Sasaki et al. [3] grew $\sim 1000$ monolayer (ML) thick AlAs/GaAs d-SL's (i.e., $A=$ AlAs, $G=$ GaAs) with $n$ and $m$ chosen from a set of small integers $\{1,2,3\}$, viz., $p_{A}=p_{G}=p$, with $p(1)=p(2)=p(3)=\frac{1}{3}$. Since the average layer thickness is 2 monolayers, one can think of this d-SL as evolving from an $(A)_{2} /(G)_{2}$ o-SL by random substitution of $A_{2}$ and $G_{2}$ layers by $A_{1}, A_{3}, G_{1}$, and $G_{3}$ layers (" $\delta$-doping"). Such disordered superlattices have shown surprising and unique optical properties relative to their parent o-SL [3]: (a) strong and initially fast decaying (lifetime $\tau=0.25$ ns at $T=77 \mathrm{~K}$ ) photoluminescence (PL) intensity even though the equivalent o-SL has an indirect band gap and thus emits both weakly and slowly, (b) a large red shift $(\sim 60 \mathrm{meV})$ of the PL peak with respect to the equivalent o-SL, and (c) an order of magnitude slower rate of reduction of the PL intensity with temperature. These unusual properties of d-SL's appear very attractive for optoelectronic applications [3].

In modelling the electronic structure of a d-SL [2,66,7], one faces difficulties arising from the existence of two entirely different length scales: (i) The lack of translational symmetry requires the use of unit cells with a macroscopic length $N \approx 1000 \mathrm{ML}$, equal to the total length of the d-SL $(N d \approx 300 \mathrm{~nm}$, where $d$ is the monolayer thickness). (ii) The spatial variations of the electron potential, however, occur on a microscopic length scale of about $0.1 \mathrm{~nm}$. While it is possible to rescale the microscopic length scale by replacing the periodic atomic potential by an external, rectangular potential [2,6], this approach fails to describe the band structure (e.g., the indirect gap of the $(\mathrm{AlAs})_{2} /(\mathrm{GaAs})_{2}$ o-SL) in the present 
regime of rapid layer fluctuations. To overcome the problems arising from the existence of two disparate length scales, we extended a microscopic pseudopotential description of the electron structure to a macroscopic length scale necessitated by the absence of translational symmetry. We use fixed (screened) atomic pseudopotentials that were carefully fitted to bulk band structures, effective masses, deformation potentials, band offsets, and energy levels in superlattices [8]. The wavefunctions are expanded in about 30 plane waves per atom. For an $\mathrm{N}$-monolayer superlattice along (001) with two atoms per monolayer the corresponding matrix dimension is therefore about $60 N \times 60 N$. Standard techniques to solve the Schrödinger equation require orthogonalization of the states of interest (i.e., near the band gap) to all lower-lying states. This leads to an $N^{3}$ scaling of the effort and becomes impractical for structures with $N \approx 100$ ML. We use instead the "folded-spectrum" method [9], where eigenstates are obtained directly in an energy window of interest (e.g., near the band gap), without having to solve for any of the $\sim 8 N$ lower-lying eigenstates first, thus circumventing the need for orthogonalization. The effort scales linearly with $N$, allowing us to use the realistic, three-dimensional pseudopotentials, and to solve the Schrödinger equation in a highly flexible plane-wave basis even for $N=1000 \mathrm{ML}$.

Application to d-SL's of AlAs/GaAs leads to an explanation of the large red shift, the enhanced oscillator strength, and the weak temperature dependence in terms of band-edge wavefunction localization. The source of localization is interesting: In truly one-dimensional disordered chains all states are in general localized [4, [0]. However, the laboratory-grown [3] d-SL's have extended layers in the $(x, y)$ plane (perpendicular to the disorder direction $z=$ [001]), so these quasi one-dimensional systems retain in fact a three-dimensional character, and the states need not be localized by disorder. We will show that the localization in the d-SL originates mostly from the formation of impurity-like, localized bound states due to insertion of $\delta$-layers into the o-SL.

In Fig. 1(a) we show the planar wavefunction average $\left|\bar{\psi}_{E}(z)\right|^{2}=\int d^{2} r_{\perp}\left|\psi_{E}(\boldsymbol{r})\right|^{2}$ of a few occupied and unoccupied band-edge states of a d-SL with a 1000-monolayer unit cell. We see that these band-edge wavefunctions are sharply localized. We can quantify the effective 
localization length (in monolayer units) for wavefunction $\psi_{E}$ at energy $E$ as [5]

$$
L_{\mathrm{eff}}(E)=\frac{1}{h}\left(\sum_{i}\left|\left\langle i \mid \bar{\psi}_{E}\right\rangle\right|^{4}\right)^{-1},
$$

where the sum extends over the grid points $i$ along $z$, and $h$ is the number of grid points per monolayer. For a truly extended state, $L_{\text {eff }}$ is of the order of $N$, while for a state localized on one site, $L_{\text {eff }}=1$. We find that $L_{\text {eff }} \lesssim 15 \mathrm{ML}$ for the band-edge states. The asymptotic decay length $\gamma^{-1}$ can be quantified by $\left\langle\left|\bar{\psi}_{E}(z)\right|\right\rangle \propto e^{-\gamma z}$, where the angular brackets denote averaging over the fast oscillations of $\left|\bar{\psi}_{E}(z)\right|$ along $z$. Near the band edges, the calculated values are $\gamma \approx 0.2 \mathrm{ML}^{-1}$.

We now investigate two possible mechanisms for the wavefunction localization apparent in Fig. 1(a): (i) chemical binding to $\delta$-like "impurity layers" in an otherwise ordered $(A)_{2} /(G)_{2}$ host, and (ii) a continuous increase of localization (measured by $\gamma$ or by $L_{\text {eff }}^{-1}$ ) with increasing degree of disorder. Scenario (i) is motivated by the fact that in one dimension an attractive $\delta$-potential always has one bound state, whereas scenario (ii) is valid for the Anderson model with on-site disorder obeying a continuous probability distribution [5]. In our case of a discrete probability distribution $p(n)$, we connect the two pictures by starting with the reference o-SL $(A)_{2} /(G)_{2}$, and gradually substituting $A_{2}$ and $G_{2}$ layers by "wrong" layers with $n=1$ or $n=3$. We measure the degree of disorder by counting the relative frequency $R$ of these layers, i.e.,

$$
R=p(1) / p(2)=p(3) / p(2)
$$

The fully disordered SL has $R=1$, the single $\delta$-layer in an ordered $(A)_{2} /(G)_{2}$ superlattice corresponds to $R \approx N^{-1}$, while the perfect o-SL has $R=0$.

To understand the possibility of impurity-like localization, consider for example a $G_{3} \delta$ layer embedded in the otherwise perfect o-SL $\cdots A_{2} G_{2} A_{2} G_{2} A_{2} G_{2} \cdots$, thus converting it into $\cdots A_{2} G_{2} A_{2} G_{3} A_{2} G_{2} \cdots$. If the $G_{3} \delta$-layer is attractive to electrons (holes) it will bind a state below the conduction-band minimum (CBM) [above the valence-band maximum (VBM)] of the o-SL 10]. We find that a $(\mathrm{GaAs})_{3} \delta$-layer in the $(\mathrm{AlAs})_{2} /(\mathrm{GaAs})_{2} \mathrm{o}-\mathrm{SL}$ indeed binds 
an electron and a hole [Fig. 1(b)], while an (AlAs) $)_{3}$ layer binds an electron but does not bind a hole [Fig. 1(c)]. The bound-state localization lengths $\gamma^{-1}$ and $L_{\text {eff }}$ obtained with a single $\delta$-layer are similar to those obtained in the fully disordered SL (Fig. 1), suggesting that the same mechanism of localization could be at work in both cases. As one increases the concentration $R$ of randomly positioned $\delta$-layers, one finds more bound states which are eventually forming a quasi continuum inside the band gap [11]. This is illustrated in Fig. 2, where the band-edge energies of d-SL's with $N=128 \mathrm{ML} 12$ are plotted as a function of the degree of disorder $R$. As $R$ approaches zero, the band edges merge with the finite binding energies of an isolated $\delta$-layer [scenario (i)], and not with the unperturbed band edges of the o-SL [scenario (ii)]. This significant observation suggests that the localization energy in the d-SL comes mostly from impurity binding. Figure 2 shows that for large degrees of disorder $R$, the band edges are pushed further into the gap. To isolate the effect of pure disorder from the effect of impurity-like localization, we also show in Fig. 2 the band edges of an o-SL containing a periodic array of $\delta$-layers of concentration $R$, i.e., separated by a distance $\sim R^{-1}$. We see that even for an array of closely spaced $\delta$-layers $(R \rightarrow 1)$, the binding energy does not increase, indicating a negligible interaction between the neighboring, coherently arranged bound states. In contrast, in a d-SL the $\delta$-layer-like bound states are arranged incoherently, leading to a band tail. These studies show that the localization length of the band-edge states in a d-SL is decided already by the chemical, impurity-like binding of a single $\delta$-layer [Figs. 11(b) and 1(c)]. Furthermore, the energy position of the gap levels at small to intermediate degrees of disorder $R$ is also determined by the properties of noninteracting, periodically arranged $\delta$-layers (Fig. 2). For higher values of $R$, disorder shifts and broadens the gap levels further into the gap, without modifying their localization length.

To illustrate the dependence of the gap-level shifts on the particular form of disorder, we compare the results obtained for the d-SL with those found in a partially-ordered superlattice (po-SL): Arent et al. [13] grew po-SL's with the same distribution function $p(n)$ for the $A_{n}$ layers as in a d-SL, but preserved long-range order by requiring that each $A_{n}$ layer be followed by a $G_{4-n}$ layer. Therefore, at positions $1,5,9, \ldots$ there is always an $A$ layer, and at 
positions $4,8,12, \ldots$ there is always a $G$ layer, while at the "sandwiched" positions, $A$ and $G$ layers are distributed randomly. We see in Fig. 2 that the presence of long-range order leads to an even larger shift of gap levels than in the equivalent d-SL at the same $R$ value. The band-edge wavefunctions have the same characteristic $L_{\text {eff }}$ and $\gamma$ as in a d-SL. Thus, absence of long-range order (in the d-SL) is not essential for obtaining large band-edge shifts.

The calculated band-gap reduction of the d-SL and po-SL with respect to the o-SL (Fig. 2) is consistent with experiment [3, 13]: We find band gaps of 2.09, 1.94, and $1.87 \mathrm{eV}$ for the o-SL, d-SL, and po-SL, respectively, compared with the experimental PL emission lines at 2.02, 1.96 [3], and $1.87 \mathrm{eV}$ [13], respectively.

To determine if the d-SL has a direct or indirect gap in the two-dimensional Brillouin zone, we show in Figure 3 the dispersion of the band-edge states of the d-SL (solid lines), o-SL (thin lines) and single $\delta$-layer (dotted lines) along the symmetry lines $\bar{\Sigma}$ and $\bar{\Delta}$, i.e., from $\bar{\Gamma}$ to $\bar{M}=\frac{1}{\sqrt{2}}(1,1)$ and from $\bar{\Gamma}$ to $\bar{X}=\frac{1}{\sqrt{2}}(1,0)$, respectively. The thin horizontal lines denote the band edges of the underlying o-SL. We find that the conduction bands of the d-SL dip below these lines. The difference ("binding energy") increases in the order $\bar{M} \rightarrow \bar{\Gamma} \rightarrow \bar{X}$. [14] Thus, the large binding energy at $\bar{\Gamma}$ pulls the conduction-band edge below the one at $\bar{M}$ by $60 \mathrm{meV}$, making the $d$-SL a direct-gap material [15], even though the o-SL is indirect (with CBM at $\bar{M}$ ). This suggests strongly that the observed [3] strong PL intensity is due to the occurrence of a direct band gap, leading to efficient recombination of electrons and holes localized in the same spatial region along $z$ [see, e.g., the same positions along the chain of states v2 and c3 in Fig. [1(a)]. The localization along $z$ relaxes the $\boldsymbol{k}_{\|}$-selection rule, thus further enhancing the oscillator strength. The enhanced oscillator strength is reflected by short radiative lifetimes $\tau$ : we calculate $\tau=1$ ns for the $\mathrm{v} 2 \rightarrow \mathrm{c} 3$ transition at energy 1.96 eV [Fig. [1(a)]. These radiative lifetimes are $1000 \times$ faster compared to those measured in indirect-gap o-SL's $(\tau \approx 5.5 \mu$ s at $T=2 \mathrm{~K})$ [16].

To understand why the PL intensity in a d-SL has a weaker temperature dependence than in an o-SL, consider vertical transport to non-radiative recombination centers. This channel will be inhibited, unless there are strongly overlapping electron states within an 
energy $\sim k T$ of each other. Strong overlap occurs when $\zeta(E, T)=k T \rho\left(E_{T}\right) L_{\text {eff }}\left(E_{T}\right)>1$, where $\rho(E)$ is the one-dimensional density of states, and $E_{T}$ is a typical energy that is thermally occupied at temperature $T$. Figure 4 shows $\rho(E), L_{\text {eff }}(E)$, and $\zeta(E, T)$ obtained by averaging over 100 realizations of $N=2000$ ML d-SL's, calculated within the KronigPenney approximation with band offset fitted to our pseudopotential calculations. We find that: (i) the DOS exhibits a $\sim 200 \mathrm{meV}$ wide band tail extending below the CBM of the o-SL (the zero of energy); (ii) $L_{\text {eff }}$ is almost constant ( $\left.\sim 20 \mathrm{ML}\right)$ in the band tail; (iii) at the thermally populated levels [denoted by the arrows in Fig. $⿴ 囗 十$ (b)] $\zeta \ll 1$, thus vertical hopping is suppressed. Consequently, the d-SL will have a weaker temperature dependence of the PL decay, because higher temperatures will be needed to dissociate the electron-hole pairs in the vertical dimension.

Acknowledgment-Fruitful discussions with D. J. Arent and S.-H. Wei are gratefully acknowledged. This work was supported by the Office of Energy Research, Materials Science Division, U.S. Department of Energy, under grant No. DE-AC36-83CH10093. 


\section{REFERENCES}

[1] J. L. Beeby et al. (editors), Condensed Systems of Low Dimensionality, NATO ASI Series B 253, (Plenum Press, New York, 1991).

[2] A. Chomette et al., Phys. Rev. Lett. 57, 1464 (1986).

[3] A. Sasaki et al., Jpn. J. Appl. Phys. 28, L1249 (1989); J. Crystal Growth 115, 490 (1991).

[4] R. E. Borland, Proc. R. Soc. A 274, 529 (1963).

[5] C. Papatriantafillou and E. N. Economou, Phys. Rev. B 13, 920 (1976).

[6] L. Pavesi et al., Phys. Rev. B 39, 7788 (1989).

[7] E. G. Wang et al., Appl. Phys. Lett. 64, 443 (1994).

[8] K. A. Mäder and A. Zunger, Phys. Rev. B, BT5032, in press (1994).

[9] L.-W. Wang and A. Zunger, J. Chem. Phys. 100, 2394 (1994).

[10] K. A. Mäder and A. Baldereschi, Mat. Res. Soc. Symp. Proc. 240, 597 (1992).

[11] I. M. Lifshits, Sov. Phys. JETP 17, 1159 (1963).

[12] Note that in order to correctly describe localized wavefunctions we need $N \gg L_{\text {eff. }}$ We have confirmed that band-edge wavefunctions calculated in an $N=128 \mathrm{~d}$-SL agree very well with those shown in Fig. 1(a), which were calculated with $N=1000$.

[13] D. J. Arent et al., Phys. Rev. B 49, 11173 (1994).

[14] The large binding energy at $\bar{X}$ is a consequence of the level repulsion of the folded $L_{1 \mathrm{c}}$ states, which is much stronger for odd values of the repeat period $n$ than for even $n$ [See, for example, S.-H. Wei and A. Zunger, Appl. Phys. Lett. 53, 2077 (1988)]. In the d-SL the odd-even selection rule is broken, leading to a stronger level repulsion in the d-SL than in the $n=2$ o-SL. 
[15] In a recent tight-binding study of an $N=10-20 \mathrm{ML}$ model of a d-SL, Wang et al. [7] found a nearly dispersionless conduction band along $\bar{\Delta}$ and $\bar{\Sigma}$, an indirect gap at $\bar{M}$, and a 2-4 ML localization length. The differences with respect to the present pseudopotential calculation may reflect a combination of the use of rather short SL's and the restricted variational flexibility of the tight-binding method used in Ref. [7].

[16] W. Ge et al., J. Luminesc. 59, 163 (1994). 


\section{FIGURES}

FIG. 1. Planar average of the wavefunctions squared in (a) a 1000-monolayer d-SL, (b) an o-SL host containing a single (GaAs) ${ }_{3} \delta$-impurity and (c) a single $(\mathrm{AlAs})_{3} \delta$-impurity. Unoccupied states are labeled $\mathrm{c} 1, \mathrm{c} 2, \ldots$; occupied states are labeled $\mathrm{v} 1, \mathrm{v} 2, \ldots$, and are plotted in the negative direction with a small offset for clarity. $L_{\text {eff }}$ and $\gamma$ are given in $\mathrm{ML}$ and $\mathrm{ML}^{-1}$, respectively.

FIG. 2. Band-edge energies of disordered (d-SL, diamonds), partially ordered superlattices (po-SL, pluses), and a periodic array of $\delta$-layers embedded in an o-SL (thin horizontal lines) as a function of $R$ [Eq. (2)]. Lines are guides to the eye. The thick horizontal lines denote the unperturbed band edges of the parent o-SL. The vertical bars on the d-SL data points denote the range of binding energies obtained from $\sim 10$ different realizations of a d-SL with length $N=128$.

FIG. 3. Dispersion of the band-edge state in a d-SL (solid lines), o-SL (thin lines), and a single $\delta$-layer in an o-SL host (dotted lines) along the $\bar{\Delta}$ and $\bar{\Sigma}$ symmetry lines in the planar Brillouin zone. Also shown are the (001)-projected bands of the o-SL (shaded area). The supercell size was $N=128$.

FIG. 4. (a) Density of states $\rho(E)$ (solid line), $L_{\text {eff }}(E)$ (dashed line) averaged over 100 realizations of $N=2000$ ML d-SL's, calculated with the Kronig-Penney model. Dots indicate $L_{\text {eff }}(E)$ obtained for an $N=128 \mathrm{ML}$ pseudopotential calculation. (b) The product $\zeta(E, T)=k T \rho(E) L_{\mathrm{eff}}(E)$. 


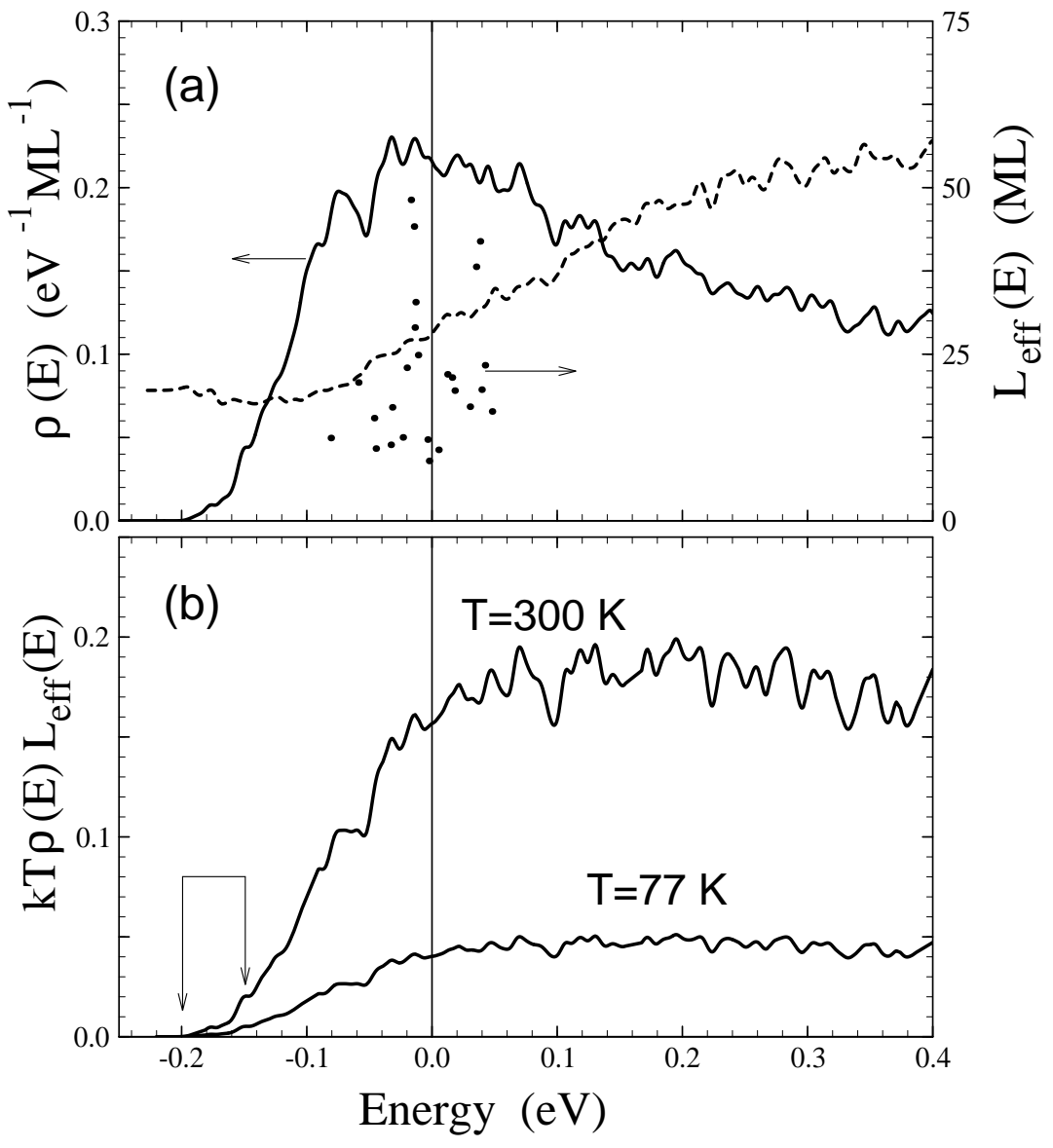

Figure 4 (revised), Mader and Zunger 


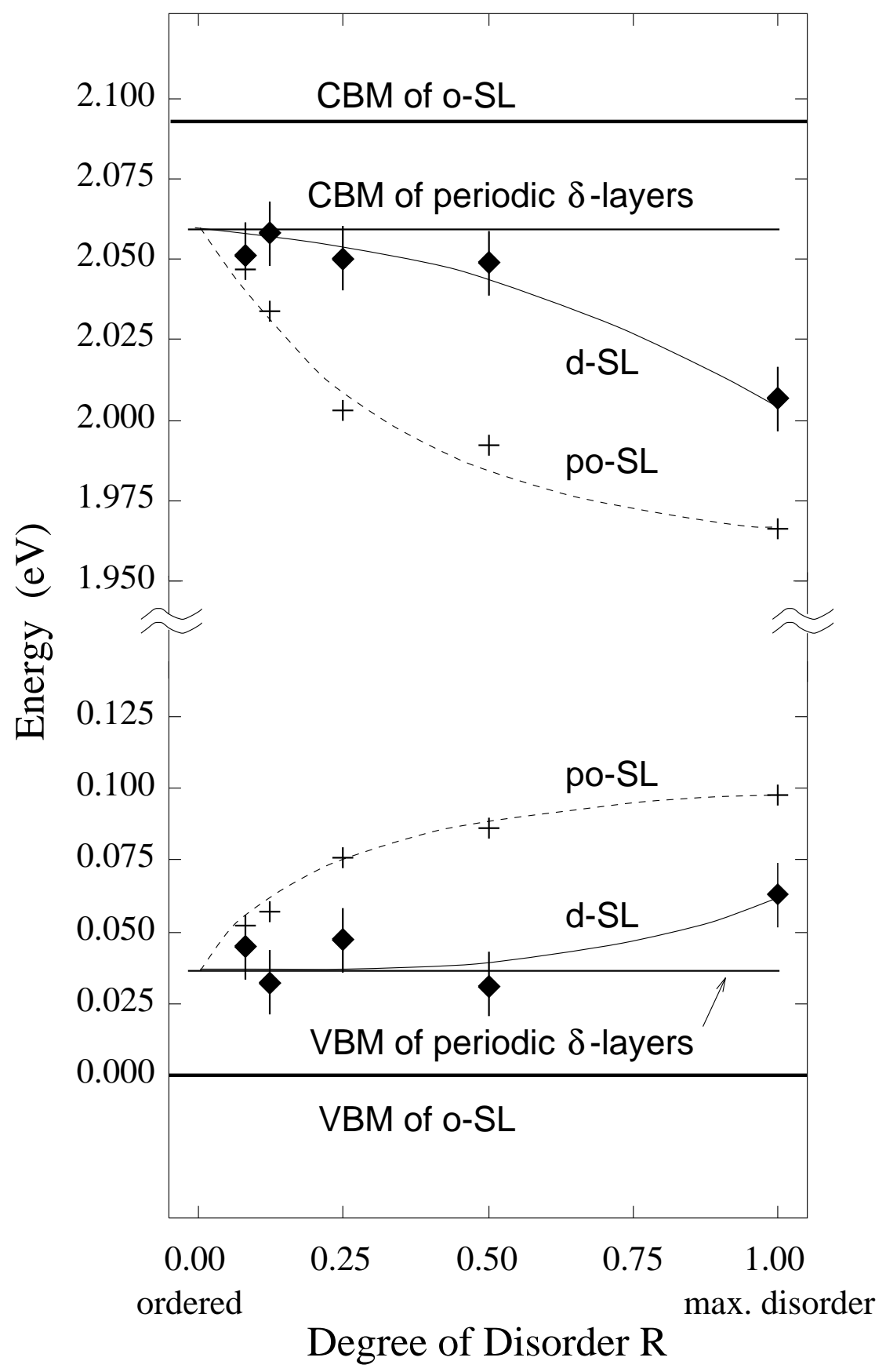

Figure 2 


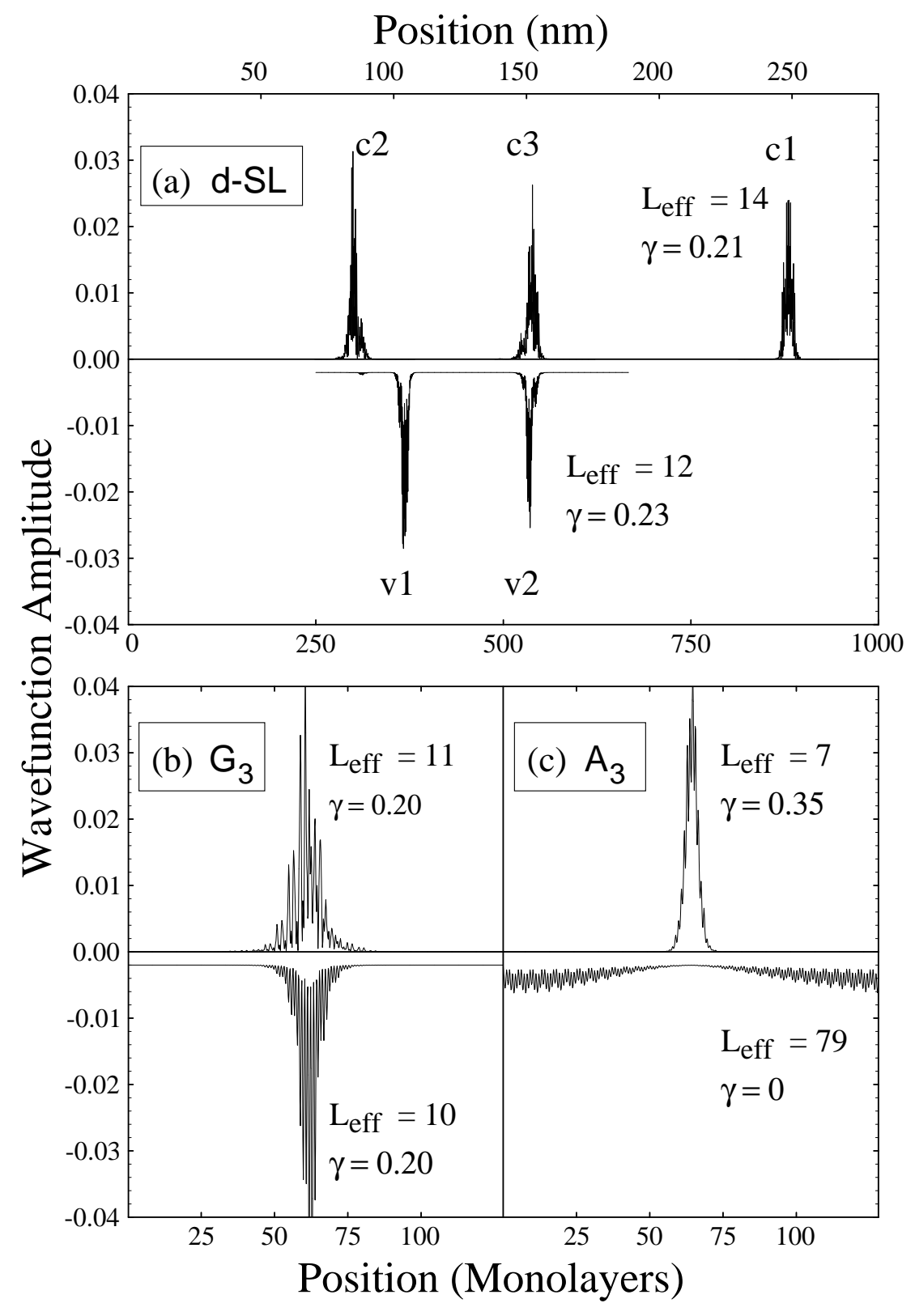

Figure 1 


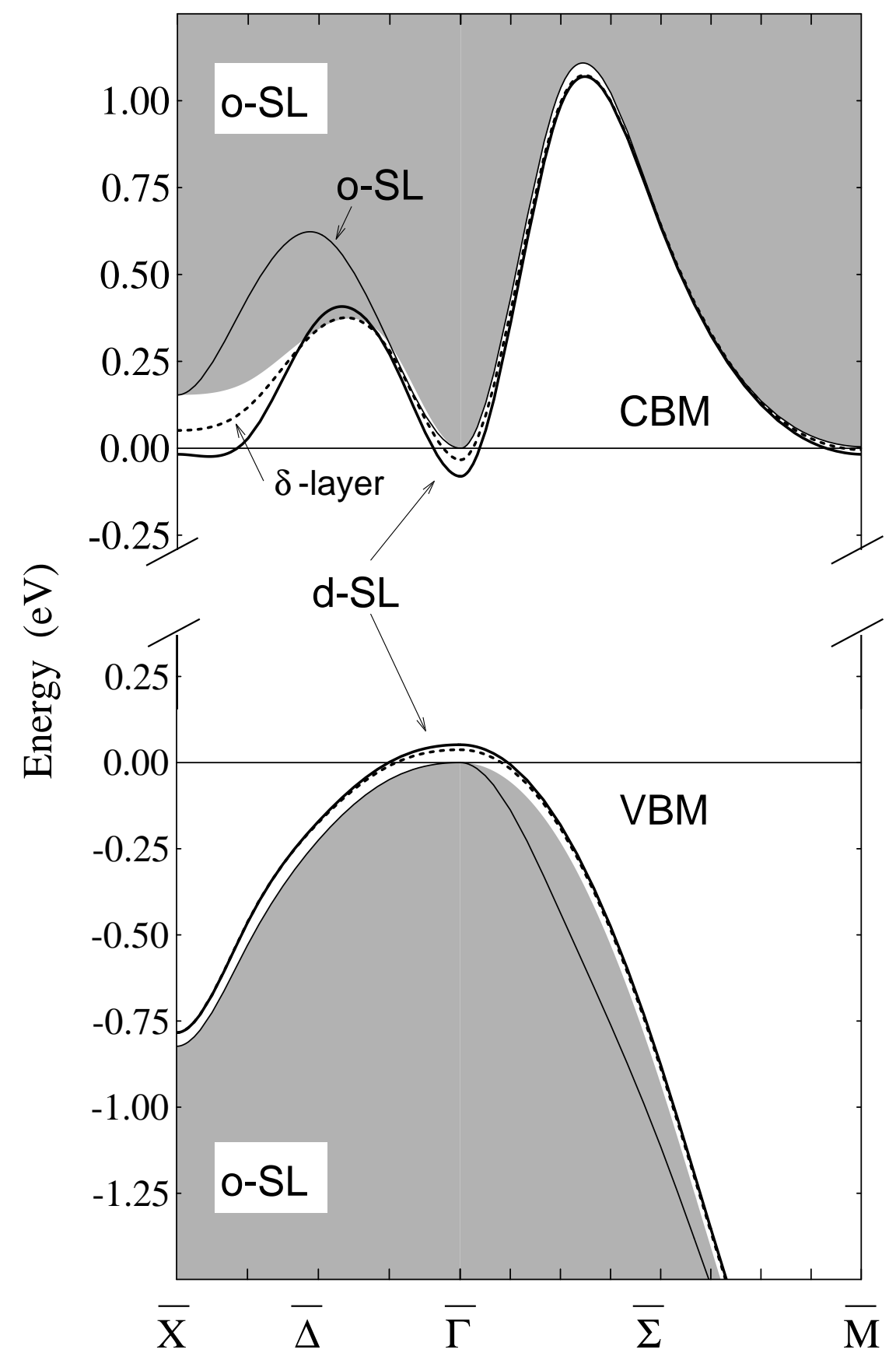

Figure 3 